\title{
Two-stage convolver-based DCT and IDCT algorithms
}

J.-L. Wu

S.-H. Hsu

W.-J. Duh

Abstract: Discrete cosine transform is the most popular solution for image coding owing to its near optimal performance, yet it is not signal dependent. A two-stage convolver-based DCT and IDCT algorithm with power-of-two length is presented. The transform matrix of IDCT is decomposed into the product of pre- and post processing matrices by using Möbius inversion formula. The pre-processing stage consists of just additions and subtractions; the post-processing stage performs circular convolution operations. A nearly recursive computational method for preprocessing calculation is given which considerably reduces the operation count and is valuable in practical usage. The post-processing is a circular convolution and can be computed using number theoretic transform, (semi-) systolic arrays or transversal filters.

\section{Introduction}

Discrete orthogonal transforms are increasingly important in many practical applications, such as data compression and spectrum analysis [1]. Among these various discrete orthogonal transforms, the discrete cosine transform (DCT) is generally recognised as the best solution to encode digital speech and image owing to its near optimal performance, yet it is not signal dependent [2]. Along with the growing applications of DCT, many fast algorithms had been proposed [3-7]. Ersoy [8] proposed a two-stage algorithm for DFT which is based on the vector transformation of sines and cosines into new basis functions using the Möbius inversion of number theory [9].

A new IDCT algorithm, with power-of-two length, derived following the construction method developed in Reference 8 , is proposed. The kernel matrix of the transform is factorised into pre-processing and post-processing matrices. The pre-processing stage consists of just additions and subtractions. The post-processing stage is a circular-convolver and can be realised using contemporary finite impulse response (FIR) filter implementation techniques. Although the development of the proposed algorithm is similar to that of Reference 8 , the resultant

Paper 8334I (E4), received 31st May 1991

The authors are with the Department of Computer Science and Information Engineering, National Taiwan University, Taipei, Taiwan Republic of China two-stage convolver-based IDCT algorithm is thought to be new.

\section{Preliminaries for two-stage IDCT algorithm}

Since the IDCT can be realised simply by transposing the signal flowgraph of the DCT and vice versa, it is sufficient to consider the IDCT-like equation

$$
x(k)=\sum_{n=0}^{N-1} \hat{y}(n) \cos \left(\frac{\pi(2 k+1) n}{2 N}\right)
$$

where

$$
\begin{aligned}
& \hat{y}(0)=\frac{1}{\sqrt{ }(2)} y(0) \\
& \hat{y}(n)=y(n) \quad n=1,2, \ldots, N-1
\end{aligned}
$$

and $N=2^{m}$, where $m$ is an integer

The sequence $x(k)$ in eqn. 1 is first partitioned into odd and even sequences which then forms a new sequence $z(k)$ $[4]$ as

$$
\begin{aligned}
z(k) & =x(2 k) \\
z\left(k+\frac{N}{2}\right) & =x(N-(2 k+1))
\end{aligned}
$$

where $k=0,1, \ldots, N / 2-1$

Thus from eqns. 1 and 3

$$
z(k)=\sum_{n=0}^{N-1} \hat{y}(n) \cos \left(\frac{\pi(4 k+1) n}{2 N}\right)
$$

where $k=0,1, \ldots, N-1$. Eqn. 1 can now be presented in another form as

$$
\tilde{z}(k)=\sum_{n=0}^{N-1} \hat{y}(n) \cos \left(\frac{\pi k n}{2 N}\right)
$$

where $\tilde{z}(4 k+1)=z(k)$.

For convenience, an index group $I(4 N)$ is defined as

$I(4 N)=4 k+1 \bmod 4 N$

where $k$ is an integer.

From the relationship between $\tilde{z}(k)$ and $z(k)$, it is clear that the evaluation of $\tilde{z}(k)$ on the index group $l(4 N)$ results in $z(k)$.

According to the derivation in Reference 8 , the cosine function can be represented in another set of basis, say 
$b\left(m_{1}, N\right)$, by using the Möbius inversion formula [9] as

$$
\begin{aligned}
\cos \left(\frac{2 \pi n}{N}\right) & =\sum_{m_{1}=1}^{M} b\left(m_{1}, N\right) u\left(\frac{m_{1} n}{N}+\frac{1}{4}\right) \\
u(x) & =\left\{\begin{array}{rl}
1 & 0<x \bmod 1<0.5 \\
-1 & 0.5<x \bmod 1<1 \\
0 & x \bmod 1=0 \text { or } 0.5
\end{array}\right.
\end{aligned}
$$

where $M=N-3$ and $m_{1} \in I(N)$ as $N$ is a power-of-two. Substituting eqn. 6 into eqn. 5

$$
\begin{aligned}
& \tilde{z}(k)=\sum_{n=0}^{N-1} \tilde{y}(n) \sum_{m_{1}=1}^{M} b\left(m_{1}, 4 N\right) u\left(\frac{m_{1} n k}{4 N}+\frac{1}{4}\right)
\end{aligned}
$$

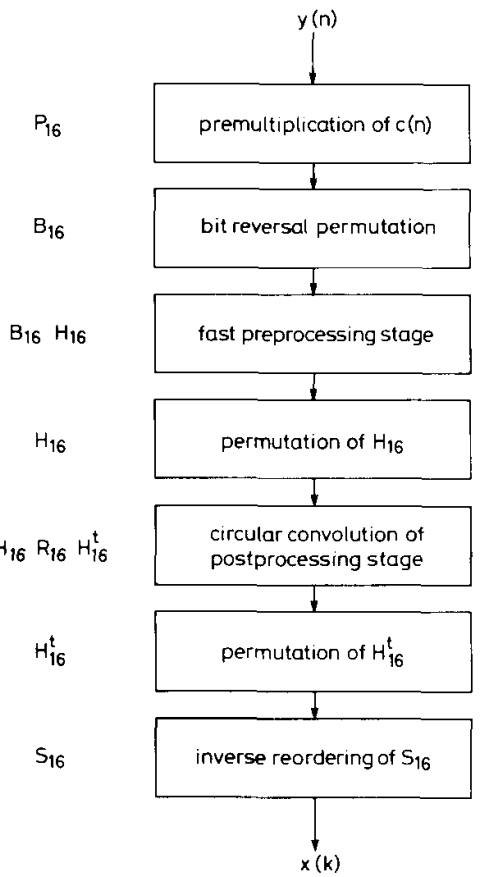

Fig. 1 System block diagram of proposed IDCT $N=16$

where $M=4 N-3$ and $m_{1}, k \in I(4 N)$. Interchanging the order of the summation, eqn. 7 can be decomposed into two stages

$$
\begin{aligned}
& h(l)=\sum_{n=0}^{N-1} \hat{y}(n) u\left(\frac{n l}{4 N}+\frac{1}{4}\right) \\
& \tilde{z}(k)=\sum_{m_{1}=1}^{M} b\left(m_{1}, 4 N\right) h\left(m_{1} k \bmod 4 N\right)
\end{aligned}
$$

In eqn. 8 , the sequence $\hat{y}(n)$ is transformed through the pre-processing stage to an intermediate sequence $h(l)$; in eqn. 9 , the post-processing stage, $h(l)$ is transformed to the sequence $\tilde{z}(k)$.

Since $k$ and $m_{1}$ of eqn. 9 are both in $I(4 N)$, it is easy to find a primitive root [9] of order $N$ to generate a new sequence such that it is in a circular convolution form. Let $g$ be a primitive root of order $N$ of the index group $I(4 N)$, then the following index mappings are defined:

$$
\begin{aligned}
k & =g^{k^{\prime}} \bmod 4 N \\
m_{1} & =g^{s m_{1}^{\prime}} \bmod 4 N
\end{aligned}
$$

where $g$ can be $4 N-3$ and $s=+1$ or -1 . Substituting eqn. 10 into eqn. 9, the equation becomes a circular convolution as

$$
\tilde{z}\left(g^{k^{\prime}}\right)=\sum_{m_{1}=0}^{N-1} b\left(g^{s m_{i}^{\prime}}, 4 N\right) h\left(g^{k^{\prime}+s m_{i}^{\prime}} \bmod 4 N\right)
$$

where $k^{\prime}=0,1, \ldots, N-1$. As shown in eqn. 11, when $s=-1$ the post-processing becomes a circular convolution; and when $s=+1$ it is a circular correlation.

From eqns. 6 and 8 , it is clear that the pre-processing stage involves additions and substractions only; and also from eqns. 6,9 and 11 , the post-processing can be converted to a circular convolution form which can be computed by using circular-convolver [10] or NTT (number theoretic transform) [11]. Therefore, both pre- and postprocessing stages are suitable for realistic implementation

\section{Fast computations of pre-processing stage}

Following the definition of eqn. 6 , a two-stage convolverbased IDCT is expressed in eqns. 8 and 9. Although the pre-processing stage consists of additions/subtractions only, direct computation of the proposed algorithm takes about $n^{2}$ multiplications and additions. Thus for real applications, it is necessary to reduce the operation counts and modularise the computational structure. Matrix algebra is used to clarify the derivations. Representing the IDCT in matrix form as

$$
\boldsymbol{C}_{\boldsymbol{N}}=\boldsymbol{S}_{\mathrm{N}} \boldsymbol{R}_{N} \boldsymbol{Q}_{N} \boldsymbol{P}_{\mathrm{N}}
$$

where $C_{N}$ denotes the IDCT transform matrix; $P_{N}$ denotes the diagonal matrix for multiplication needed in eqn. $2 ; \boldsymbol{Q}_{N}$ is the pre-processing matrix in eqn. $8 ; R_{N}$ is the post-processing matrix in eqn. $9 ; \boldsymbol{S}_{N}$ is the inverse reordering described in eqn. 3 ; and the subscript $N$ denotes the transform length.

Decomposition of $\boldsymbol{Q}_{N}$ is the main theme. Since $l \in I(4 N)$, the symmetry of $Q_{N}$ is obtained as follows:

$$
\begin{aligned}
h\left(l^{\prime}\right)= & \sum_{n=0}^{(N / 2)-1} \hat{y}(2 n) u\left(\frac{\left(4 l^{\prime}+1\right)}{2 N}+\frac{1}{4}\right) \\
& +\hat{y}(2 n+1) u\left(\frac{(2 n+1)\left(4 l^{\prime}+1\right)}{4 N}+\frac{1}{4}\right) \\
h\left(\frac{N}{2}+l^{\prime}\right)= & \sum_{n=0}^{(N / 2)-1} \hat{y}(2 n) u\left(\frac{\left(4 l^{\prime}+1\right) n}{2 N}+\frac{1}{4}\right) \\
& +\hat{y}(2 n+1) u\left(\frac{(2 n+1)\left(4 l^{\prime}+1\right)}{4 N}+\frac{1}{2}+\frac{1}{4}\right)
\end{aligned}
$$

where $l^{\prime}=0,1, \ldots,(N / 2)-1$. Since

$$
u\left(\theta+\frac{1}{2}\right)=-u(\theta)
$$

it folows that

$$
\tilde{Q}_{N}=\left[\begin{array}{rr}
\tilde{Q}_{N / 2} & D_{N / 2} \\
\tilde{Q}_{N / 2} & -D_{N / 2}
\end{array}\right]
$$

where $\tilde{Q}_{N}=Q_{N} B_{N}$ and $B_{N}$ is the bit reversal permutation matrix.

From eqn. 8 , the function $u\left[(n l / 4 N)+\frac{1}{4}\right]$ is, in fact, the sign function of $\cos (n l / 4 N)$. To implement eqn. 16, a fast computational method devised to construct the recurrent

IEE PROCEEDINGS-I, Vol. 138, No.6, DECEMBER 1991 
relation between $D_{N / 2}$ and $\Phi_{N / 2}$ is presented as follows:

$$
D_{N / 2}=M_{N / 2} \tilde{Q}_{N / 2}+A_{N / 2}
$$

and eqn. 16 becomes

$$
\begin{aligned}
\tilde{Q}_{N}= & {\left[\begin{array}{ll}
\boldsymbol{I}_{N / 2} & \boldsymbol{I}_{N / 2} \\
\boldsymbol{I}_{N / 2} & -\boldsymbol{I}_{N / 2}
\end{array}\right]\left(\left[\begin{array}{ll}
\boldsymbol{I}_{N / 2} & \mathbf{0}_{(N / 2)} \\
\mathbf{0}_{(N / 2)} & \boldsymbol{M}_{(N / 2)}
\end{array}\right]\left[\begin{array}{ll}
\tilde{\boldsymbol{Q}}_{N / 2} & \mathbf{0}_{N / 2} \\
\mathbf{0}_{N / 2} & \tilde{\boldsymbol{Q}}_{N / 2}
\end{array}\right]\right.} \\
& \left.+\left[\begin{array}{ll}
\mathbf{0}_{N / 2} & \mathbf{0}_{N / 2} \\
\mathbf{0}_{N / 2} & \boldsymbol{A}_{N / 2}
\end{array}\right]\right)
\end{aligned}
$$

where $\boldsymbol{M}_{N / 2}$, a diagonal matrix with elements 1 or -1 , stands for the multiplication matrix and $\boldsymbol{A}_{N / 2}$, a sparse matrix with nonzero elements 2 or -2 , denotes the compensation matrix.

From eqn. 18 , it is obvious that $\tilde{Q}_{V}$ is nearly recursively decomposable. The computational diagram of eqn. 18 is illustrated in Fig. 2. The complexity of the pro-

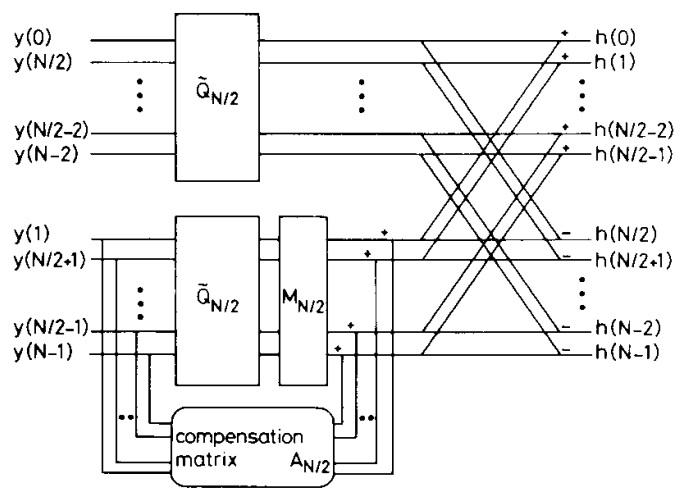

Fig. 2 Fast computation of pre-processing stage

cedure depends on the computation of matrix $A_{N / 2}$ and increases as $N$ gets larger. Though the derived recurrence is not perfect, it reduces the number of operations of the pre-processing stage. The corresponding architecture is regular and is more efficient than direct implementation of adder arrays. Table 1 shows the operation counts of some practically used lengths.

Table 1: Operation counts for the pre-processing stage

\begin{tabular}{rrrrrr}
\hline Length & $\begin{array}{l}\text { Adder array } \\
\text { Additions }\end{array}$ & \multicolumn{3}{c}{ Proposed method } & $N \log _{2} N$ \\
\cline { 3 - 5 } & & Additions & Shifts & Inversions & \\
\cline { 3 - 5 } & & 8 & 0 & 1 & 8 \\
\hline 4 & 16 & 26 & 2 & 4 & 24 \\
8 & 64 & 76 & 8 & 12 & 48 \\
16 & 256 & 236 & 30 & 32 & 160 \\
32 & 1024 & 74 & 86 & 80 & 384 \\
64 & 4096 & 748 & 80 & \\
\hline
\end{tabular}

\section{Architecture for post-processing stage}

From eqn. 11, the post-processing stage becomes a circular correlator or circular convolver depending on if $s=1$ or $s=-1$. It is well known that the circular convolution can be implemented using systolic, semi-systolic arrays and transversal filters [10]. It can also be computed by using the circular convolution property (CCP) of the NTT [11]. The post-processing stage can be converted to a circular convolution form after a simple data permutation. From the derivations in eqns. 10 and 11 , the permutation orders can be easily generated.

IEE PROCEEDINGS-I, Vol. 138, No. 6, DECEMBER 1991

\section{Discussion and conclusions}

It is clear that the post-processing stage is of circularconvolution form from the derivation of the proposed two-stage convolver-based IDCT. Since the IDCT is unitary, the DCT transform matrix is the transpose of the IDCT transform matrix. The two-stage DCT algorithm can thus be obtained by transposing the matrix $C_{N}$.

It is obviously that the pre-processing stage of the DCT is a circular convolver and can be treated as a FIR filter. The frequency response of the DCT pre-processing filter is illustrated in Fig. 3. In most of the image coding

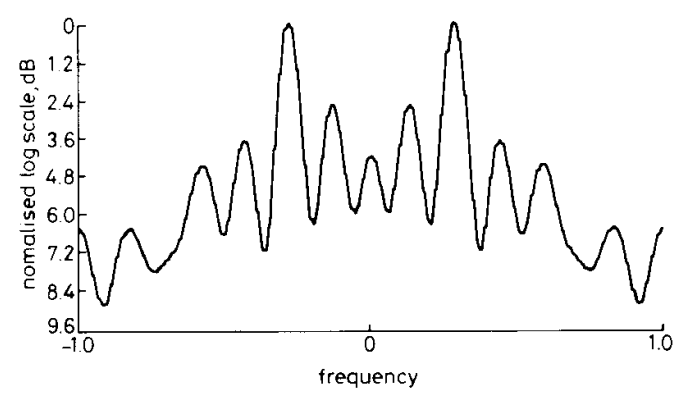

Fig. 3 Frequency response of DCT pre-processing filter

systems, a pre-filter is always used to remove high frequency noises and a post-filter, designed based on human visual properties [12], is also included to improve the picture quality, as shown in Fig. 4. Furthermore, the pre-

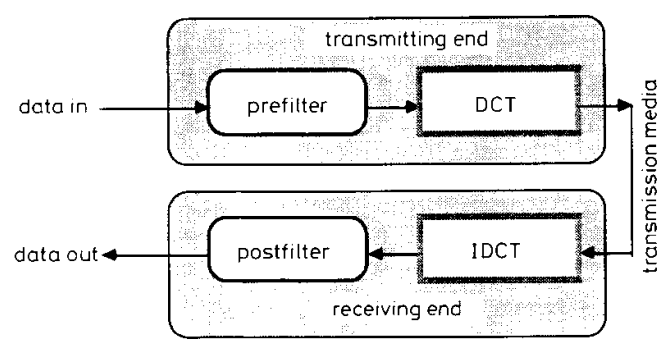

Fig. 4 Block diagram of image coding system with pre- and postfiltering

processing matrix, $Q_{N}$, can be directly applied to the field of image coding and pattern recognition $[13,14]$.

\section{References}

1 AHMED, N., and RAO, K.R.: 'Orthogonal transforms for digital signal processing' (Springer-Verlag, Berlin, 1975)

2 AHMED, N., NATARAJAN, T., and RAO, K.R.: 'The discrete AHMED, N., NATARAJAN, T., and RAO, K. 93
cosine transform', IEEE Trans., 1974, C-25, pp. 90-93

3 CHEN, W.H., SMITH, C.H., and FRALICK, S.C.: 'A fast computa3 CHEN, W.H., SMITH, C.H., and FRALICK, S.C.: 'A fast computa-
tional algorithm for the discrete cosine transform', IEEE Trans., 1977, COM-25, (9), pp. 1004-1009

4 NARASIMHA, M.J., and PETERSON, A.M.: 'On the computation of the discrete cosine transfom', IEEE Trans., 1978, COM-26, (6), pp. 934-936

5 MAKHOUL, J.: 'A fast cosine transform in one and two dimensions', IEEE Trans., 1980, ASSP-28, pp. 27-34

6 HOU, H.S. 'A fast recursive algorithm for computing the discrete 6 HOU, H.S.: 'A fast recursive algorithm for computing the discre
cosine transform', IEEE Trans., 1987, ASSP-35, pp. 1455-1461

7 LEE, B.G.: 'Input and output index mappings for a prime factored decomposed computation of discrete cosine transform', IEEE Trans., 1989, ASSP-37, (2), pp. 237--244 
8 ERSOY, O.: 'A two-stage representation of DFT and its applications', IEEE Trans., 1987, ASSP-35, pp. 825-831

APOSTOL, T.M.: 'Introduction to analytic number theory' (Springer-Verlag, New York, 1976)

10 ERSOY, O.: 'Semisystolic array implementation of circular, skew circular, and linear convolutions', IEEE Trans., 1985, C-34, (2), pp. 190-194

11 AGARWAL, R.C., and BURRUS, C.S.: 'Fast convolution using

Fermat number transforms with applications to digital filtering',

IEEE Trans., 1974, ASSP-22, pp. $87-97$
12 NGAN, K.N., LEONG, K.S., and SINGH, H.: 'Adaptive cosine transform coding of images in perceptual domain', IEEF Trans. transform coding of images in perce

1989, ASSP.37, (11), pp. 1743-1749
13 ERSOY, O.K., and KIM, D.Y.: Image recognition with the discrete

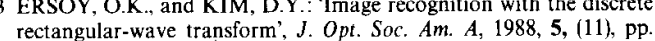
5-18

14 KIM, D.Y., and ERSOY, O.K.: 'Image recognition with the discrete rectangular-wave transform', $1989,6,(6)$, pp. 835-843 